\title{
Predictors and outcomes of sepsis-induced cardiomyopathy in critically ill patients
}

\author{
Myung Jin Song, Sang Hoon Lee, Ah Young Leem, Song Yee Kim, Kyung Soo Chung, Eun Young Kim, \\ Ji Ye Jung, Young Ae Kang, Young Sam Kim, Joon Chang, Moo Suk Park
}

Division of Pulmonology, Department of Internal Medicine, Institute of Chest Diseases, Severance Hospital, Yonsei University College of Medicine, Seoul, Korea

Background: Sepsis-induced cardiomyopathy (SIC) occurs frequently in critically ill patients, but the clinical features and prognostic impact of SIC on sepsis outcome remain controversial. Here, we investigated the predictors and outcomes of SIC.

Methods: Patients admitted to a single medical intensive care unit from June 2016 to September 2017 were retrospectively reviewed. SIC was diagnosed by ejection fraction (EF) $<50 \%$ and $\geq 10 \%$ decrease in baseline EF that recovered within 2 weeks.

Results: In total, 342 patients with sepsis met the inclusion criteria, and 49 patients (14.3\%) were diagnosed with $\mathrm{SIC}$; the latter were compared with 259 patients whose EF was not deteriorated by sepsis (non-SIC). Low systolic blood pressure and increased left ventricular enddiastolic diameter (LVEDD) were identified as predictors of SIC. SIC and non-SIC patients did not differ significantly in terms of 28 -day all-cause mortality ( $24.5 \%$ vs. $26.3 \%, \mathrm{P}=0.936)$. Acute Physiology and Chronic Health Evaluation II (APACHE II; hazard ratio [HR], 1.10; 95\% confidential interval $[\mathrm{Cl}], 1.02$ to $1.18 ; \mathrm{P}=0.009$ ) and delta neutrophil index (DNI; HR, 1.02; $95 \% \mathrm{Cl}, 1.00$ to $1.08 ; \mathrm{P}=0.026$ ) were independent risk factors for 28 -day mortality with $\mathrm{SIC}$. DNI, APACHE II, and lactate were identified as risk factors for 28-day mortality in sepsis patients as a whole.

Conclusions: SIC was not associated with increased mortality compared to non-SIC. Low systolic blood pressure and increased LVEDD were predictors of SIC. High APACHE II score and elevated DNI, which reflect sepsis severity, predict 28-day all-cause mortality.

Key Words: APACHE; delta neutrophil index; left; mortality; prognosis; sepsis; ventricular dysfunction

\section{INTRODUCTION}

Sepsis is a syndrome of physiologic, pathologic, and biochemical abnormalities induced by infection that can result in multiple organ dysfunction via inflammatory cytokines, mitochondria dysfunction, and tissue hypoxia [1]. Due to specific injury of cardiomyocytes, approximately $3.8 \%-65 \%$ of patients with sepsis develop sepsis-induced cardiomyopathy (SIC) [2-7]. SIC has been defined as myocardial dysfunction characterized by decreased ejection fraction (EF) and increased left ventricular end-diastolic diameter (LVEDD) that recovers within 7-10 days [2]. Current understanding of the pathogenesis of SIC is limited, and con-

\section{Original Article}

Received: January 14, 2020

Revised: March 17, 2020

Accepted: April 7, 2020

Corresponding author

Moo Suk Park

Division of Pulmonology, Department of Internal Medicine, Institute of Chest Diseases, Severance Hospital, Yonsei University College of Medicine, 50-1 Yonsei-ro, Seodaemun-gu, Seoul 03722, Korea Tel: +82-2-2228-1955 Fax: +82-2-393-6884 E-mail: pms70@yuhs.ac

Copyright (@) 2020 The Korean Society of Critical Care Medicine

This is an Open Access article distributed under the terms of Creative Attributions Non-Commercial License (https:// creativecommons.org/li-censes/by-nc/4.0/ which permits unrestricted noncommercial use, distribution, and reproduction in any medium, provided the original work is properly cited. 
flicting results remain regarding the prognostic impact of SIC on sepsis outcomes.

Improved understanding of SIC is important for multiple reasons. First, cardiac function is crucial for maintaining hemodynamic stability in patients with septic shock. Second, by understanding the clinical features and predictors of SIC, we can discriminate SIC from other cardiac diseases and avoid unnecessary invasive procedures, such as coronary angiography, a risky procedure in critically ill patients. Thus, we aimed to define clinical predictors of SIC and assess the clinical course and outcome of SIC in patients with sepsis.

\section{MATERIALS AND METHODS}

\section{Study Population}

In this study, the medical records of patients who were admitted to the medical intensive care unit (ICU) of Yonsei University College of Medicine from June 2016 to September 2017 were reviewed. A total of 576 adult patients ( $>18$ years old) admitted to the ICU during this period were screened for inclusion (Figure 1). Patients who did not (1) meet the sepsis definition, (2) have baseline transthoracic echocardiography (TTE) data, or (3) undergo TTE within 48 hours after ICU admission were excluded. This study was approved by the Insti-

\section{KEY MESSAGES}

- Sepsis-induced cardiomyopathy (SIC) occurred in 14.3\% of intensive care unit patients with sepsis.

- Low systolic blood pressure and increased left ventricular end-diastolic diameter were predictors of SIC.

- High Acute Physiology and Chronic Health Evaluation II and elevated delta neutrophil index were risk factors of 28-day mortality in SIC.

tutional Review Board and Ethics Committee of Severance Hospital (IRB No. 4-2018-0751). The requirement for written informed consent from patients was waived. All methods were performed in accordance with the Declaration of Helsinki.

\section{Definitions of Variables}

The sepsis-3 definition was applied in patients with sepsis and septic shock [1]. Sepsis was defined by an increase in Sequential Organ Failure Assessment (SOFA) score of $\geq 2$ points due to infection. Infection was defined as detection of microorganisms in culture or as radiologic or clinical manifestations suggesting infection despite negative culture results [8]. Septic shock was defined as sepsis with persistent hypotension re-

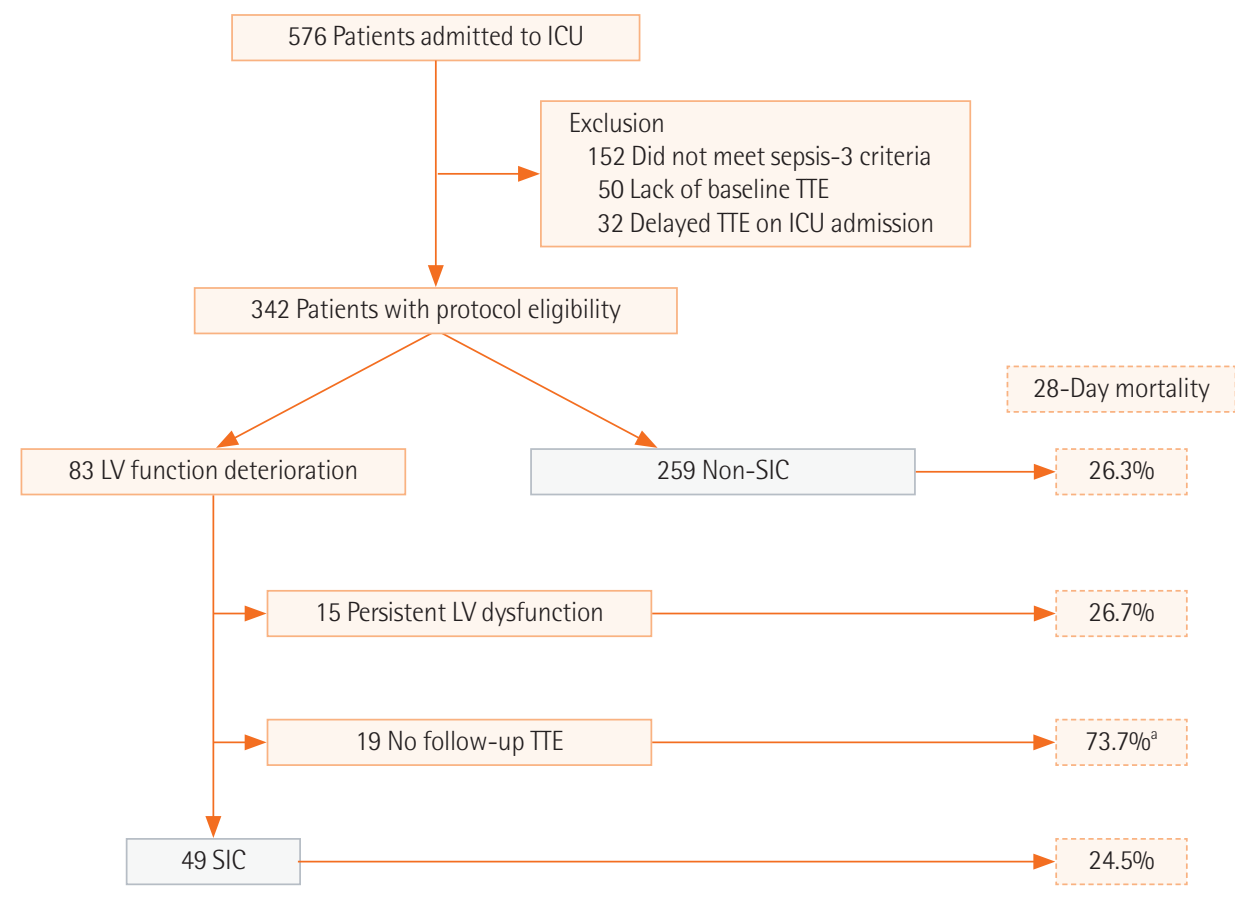

Figure 1. Patient recruitment flowchart. ICU: intensive care unit; TE: transthoracic echocardiography; LV: left ventricle; SIC: sepsis-in-

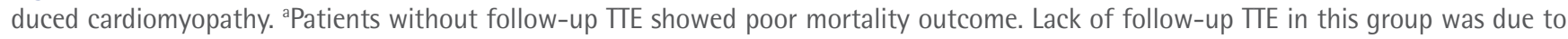
several reasons: eight patients (42.1\%) died within 72 hours after ICU admission, end-of-life decisions were made for six patients (31.6\%), two patients were transferred to other hospitals, and three patients (15.8\%) recovered from sepsis. 
quiring vasopressor drugs to maintain mean arterial pressure $\geq 65 \mathrm{~mm} \mathrm{Hg}$ accompanied with serum lactate level $>2 \mathrm{mmol} / \mathrm{L}$ despite adequate volume resuscitation. Left ventricular function deterioration was defined as $\mathrm{EF}<50 \%$ and $\geq 10 \%$ decrease in baseline EF. Among patients with left ventricular function deterioration, those whose $\mathrm{EF}$ recovered to the baseline level within 2 weeks were defined as having SIC $[7,9,10]$.

\section{Data Collection}

Demographic data (age, sex, body weight, height, pre-existing comorbidities), vital signs, laboratory findings, echocardiographic findings, and clinical findings (presence of acute kidney injury, acute respiratory distress syndrome, and use of mechanical ventilation) were collected. Laboratory tests were performed within 24 hours of ICU admission, and SOFA and Acute Physiology and Chronic Health Evaluation (APACHE) II scores were evaluated at ICU admission. The delta neutrophil index (DNI) was assessed using an automated blood cell analyzer (ADVIA 120; Siemens, Forchheim, Germany) and calculated using the following formula: (neutrophil subfraction+ eosinophil subfraction measured in the myeloperoxidase channel)-(polymorphonuclear subfraction measured in nuclear lobularity channel) $[11,12]$. If there was no contraindication, TTE was routinely performed by a cardiologist at the time of ICU admission. There was no fixed schedule for follow up TTE, but in the majority of patients it was performed 1-2 weeks after ICU admission, especially when cardiac function deteriorated during ICU admission.

\section{Statistical Analysis}

The primary endpoint was 28-day all-cause mortality of SIC. The secondary endpoint was to identify predictors of SIC development and risk factors for 28-day all-cause mortality in patients with SIC. Factors contributing to the incidence of SIC were also analyzed. Continuous variables were analyzed using Student t-test or Mann-Whitney U-test; categorical variables were analyzed using the chi-squared distribution and Fisher's exact test. A logistic regression model was used to identify variables contributing to the development of SIC. Cox proportional hazards regression analyses were conducted to assess the relationships between variables and 28-day allcause mortality. Area under the curve of receiver operating characteristic curves was used to identify the effect of major variables in multivariate Cox proportional hazards regression analysis $(\mathrm{P}<0.05)$ on 28 -day all-cause mortality. Cumulative time-to-event distribution (survival) was estimated using Kaplan-Meier survival curves, and differences in survival be- tween groups were assessed using the log-rank test. In all cases, P-values $<0.05$ were considered statistically significant. Statistical analyses were performed using R statistical software ver. 3.5.1 (R Foundation, Vienna, Austria).

\section{RESULTS}

\section{Baseline Characteristics of Study Population}

A total of 342 patients met the study eligibility criteria. Of these, 83 showed left ventricular function deterioration compared to baseline TTE. Among these, 49 patients had an EF that recovered to the baseline value and were categorized as SIC. Fifteen patients with persistently decreased EF in follow-up TTE and 19 patients without follow-up TTE were excluded (Figure 1). A total of 259 patients whose EF did not deteriorate with sepsis were categorized as the non-SIC group.

Baseline characteristics of 49 SIC and 259 non-SIC patients with sepsis are shown in Table 1 . There was no significant difference between SIC and non-SIC patients in terms of age, sex, and body mass index. APACHE II and SOFA scores also did not significantly differ between SIC and non-SIC patients. Underlying heart failure was more frequent in SIC patients ( $8.2 \%$ vs. $1.5 \%, \mathrm{P}=0.029$ ), but the incidence of other comorbidities, including coronary artery disease, was not significantly different between the groups. On ICU admission, systolic blood pressure was significantly lower in patients with SIC $(83.9 \pm 18.3$ vs. $91.1 \pm 22.9, \mathrm{P}=0.039)$. There was no significant difference in vasopressor and inotrope use between SIC and non-SIC patients. Primary site of infection was not significantly different between SIC and non-SIC patients. Both C-reactive protein and serum bilirubin levels were higher (192.4 \pm 134.2 vs. $137.5 \pm 110.6, \mathrm{P}=0.002 ; 1.2 \pm 1.4$ vs. $2.1 \pm 4.1, \mathrm{P}=0.008$, respectively), whereas serum albumin levels were lower (2.4 \pm 0.5 vs. $2.5 \pm 0.5, \mathrm{P}=0.032)$ in SIC patients than in non-SIC patients.

Echocardiographic findings and cardiac biomarkers are shown in Table 2. LVEDD and left ventricular end-systolic diameter (LVESD) were significantly increased in SIC patients (49.6 \pm 6.0 vs. $43.4 \pm 6.3, \mathrm{P}<0.001 ; 39.5 \pm 6.4$ vs. $28.7 \pm 4.8, \mathrm{P}<0.001$, respectively). N-terminal pro b-type natriuretic peptide (NTproBNP; $\mathrm{pg} / \mathrm{ml})$ and troponin-T $(\mathrm{pg} / \mathrm{ml}$ ) were significantly higher in SIC patients $(19,911.2 \pm 18,194.9$ vs. 7,940.3 $\pm 13,613.9$, $\mathrm{P}<0.001 ; 278.9 \pm 492.7$ vs. $104.8 \pm 178.4, \mathrm{P}=0.019$, respectively), but creatine kinase (CK) and creatine kinase-muscle/brain (CK-MB) levels were not significantly different between SIC and non-SIC patients. 
Table 1. Baseline characteristics of patients

\begin{tabular}{|c|c|c|c|c|}
\hline Variable & Total $(n=308)$ & $\operatorname{SIC}(n=49)$ & Non-SIC $(n=259)$ & P-value \\
\hline Age (yr) & $64.6 \pm 14.5$ & $65.1 \pm 11.2$ & $64.6 \pm 15.0$ & 0.772 \\
\hline Male sex & 195 (63.3) & $32(65.3)$ & 163 (62.9) & 0.877 \\
\hline BMI $\left(\mathrm{kg} / \mathrm{m}^{2}\right)$ & $22.2 \pm 4.3$ & $22.4 \pm 3.9$ & $22.2 \pm 4.4$ & 0.782 \\
\hline APACHE II & $24.9 \pm 8.8$ & $26.4 \pm 9.3$ & $24.6 \pm 8.6$ & 0.209 \\
\hline SOFA & $9.4 \pm 3.2$ & $9.7 \pm 3.2$ & $9.3 \pm 3.2$ & 0.378 \\
\hline \multicolumn{5}{|l|}{ Comorbidity } \\
\hline Hypertension & 159 (51.6) & $25(51.0)$ & $134(51.7)$ & 1.000 \\
\hline Diabetes mellitus & $108(35.1)$ & $17(34.7)$ & $91(35.1)$ & 1.000 \\
\hline Chronic kidney disease & $67(21.8)$ & $11(22.4)$ & $56(21.6)$ & 1.000 \\
\hline Liver cirrhosis & $25(8.1)$ & $2(4.1)$ & $23(8.9)$ & 0.399 \\
\hline Chronic liver disease & $29(9.4)$ & $1(2.0)$ & $28(10.8)$ & 0.097 \\
\hline Cancer & $83(26.9)$ & $16(32.7)$ & $67(25.9)$ & 0.420 \\
\hline Heart failure & $8(2.6)$ & $4(8.2)$ & $4(1.5)$ & 0.029 \\
\hline Coronary artery disease & $30(9.7)$ & $7(14.3)$ & $23(8.9)$ & 0.364 \\
\hline Cerebrovascular disease & $42(13.6)$ & $6(12.2)$ & $36(13.9)$ & 0.934 \\
\hline Charlson comorbidity index & $2.8 \pm 2.2$ & $3.2 \pm 2.1$ & $2.8 \pm 2.2$ & 0.231 \\
\hline Acute kidney injury & $73(23.7)$ & $14(28.6)$ & $59(22.8)$ & 0.490 \\
\hline ARDS & $28(9.1)$ & $8(16.3)$ & $20(7.7)$ & 0.099 \\
\hline Septic shock & 295 (95.8) & $49(100.0)$ & 246 (95.0) & 0.224 \\
\hline Blood culture (+) & 113 (36.7) & $22(44.9)$ & $91(35.1)$ & 0.255 \\
\hline Mechanical ventilation & $200(64.9)$ & $36(73.5)$ & $164(63.3)$ & 0.229 \\
\hline \multicolumn{5}{|l|}{ Vital sign on admission } \\
\hline $\mathrm{SBP}(\mathrm{mm} \mathrm{Hg})$ & $90.0 \pm 22.3$ & $83.9 \pm 18.3$ & $91.1 \pm 22.8$ & 0.039 \\
\hline MAP $(m m ~ H g)$ & $66.7 \pm 15.4$ & $64.3 \pm 15.3$ & $67.1 \pm 15.4$ & 0.249 \\
\hline HR (bpm) & $103.0 \pm 27.9$ & $109.6 \pm 28.9$ & $101.7 \pm 27.6$ & 0.071 \\
\hline \multicolumn{5}{|c|}{ Use of vasopressor and inotrope } \\
\hline None & $13(4.2)$ & 0 & $15(5.1)$ & 0.224 \\
\hline Norepinephrine & $262(85.1)$ & $44(89.8)$ & $247(84.3)$ & 0.420 \\
\hline Dobutamine & $3(0.9)$ & $2(4.1)$ & $1(0.4)$ & 0.105 \\
\hline Primary focus of infection & & & & 0.507 \\
\hline Pulmonary & $155(50.3)$ & $29(59.2)$ & $126(48.6)$ & \\
\hline GI tract & $43(14.0)$ & $3(6.1)$ & $40(15.4)$ & \\
\hline Urogenital & $35(11.4)$ & $7(14.3)$ & $28(10.8)$ & \\
\hline Pancreatobiliary & $25(8.1)$ & $2(4.1)$ & $23(8.9)$ & \\
\hline Soft tissue/bone & $17(5.5)$ & $4(8.2)$ & $13(5.0)$ & \\
\hline Liver & $9(2.9)$ & $1(2.0)$ & $8(3.1)$ & \\
\hline Kidney & $6(1.9)$ & $1(2.0)$ & $5(1.9)$ & \\
\hline Miscellaneous & $18(5.8)$ & $2(4.1)$ & $16(6.2)$ & \\
\hline
\end{tabular}

(Continued to the next page)

\section{Predictors of SIC}

Univariate logistic regression analyses for predictors of SIC revealed that low systolic blood pressure, hypoalbuminemia, el- evated NT-proBNP, troponin-T, and increased LVEDD were major covariates $(\mathrm{P}<0.05)$. Multivariate logistic regression analysis revealed that low systolic blood pressure upon ICU 


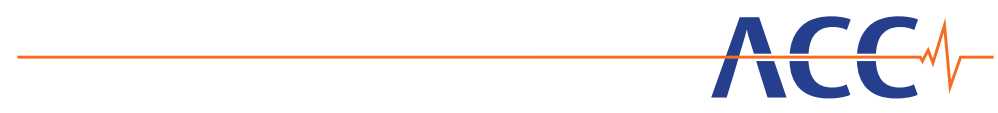

Table 1. Continued

\begin{tabular}{|c|c|c|c|c|}
\hline Variable & Total $(n=308)$ & $\operatorname{SIC}(n=49)$ & Non-SIC $(n=259)$ & P-value \\
\hline \multicolumn{5}{|l|}{ Laboratory parameter } \\
\hline $\operatorname{WBC}\left(10^{3} / \mu \mathrm{l}\right)$ & $15.1 \pm 10.4$ & $12.9 \pm 10.3$ & $15.5 \pm 10.4$ & 0.117 \\
\hline Platelet $\left(10^{3} / \mu \mathrm{l}\right)$ & $159.2 \pm 120.4$ & $162.6 \pm 139.1$ & $158.5 \pm 116.8$ & 0.831 \\
\hline Serum albumin (g/dl) & $2.5 \pm 0.5$ & $2.4 \pm 0.5$ & $2.5 \pm 0.5$ & 0.032 \\
\hline Serum bilirubin (mg/dl) & $2.0 \pm 3.8$ & $1.2 \pm 1.4$ & $2.1 \pm 4.1$ & 0.008 \\
\hline Serum creatinine $(\mathrm{mg} / \mathrm{dl})$ & $2.1 \pm 1.9$ & $2.0 \pm 1.8$ & $2.1 \pm 2.0$ & 0.757 \\
\hline Lactate (mmol/L) & $4.0 \pm 4.1$ & $3.8 \pm 3.4$ & $4.0 \pm 4.3$ & 0.826 \\
\hline CRP & $146.3 \pm 116.2$ & $192.4 \pm 134.2$ & $137.5 \pm 110.6$ & 0.002 \\
\hline Procalcitonin (ng/ml) & $17.5 \pm 33.0$ & $23.9 \pm 35.5$ & $16.2 \pm 32.4$ & 0.135 \\
\hline DNI (\%) & $11.0 \pm 15.1$ & $13.9 \pm 18.9$ & $10.5 \pm 14.2$ & 0.224 \\
\hline
\end{tabular}

Values are presented as mean \pm standard deviation or number (\%).

SIC: sepsis-induced cardiomyopathy; BMI: body mass index; APACHE: Acute Physiology and Chronic Health Evaluation; SOFA: Sequential Organ Failure Assessment; ARDS: acute respiratory distress syndrome; SBP: systolic blood pressure; MAP: mean arterial pressure; HR: heart rate; GI: gastrointestinal; WBC: white blood cell; CRP: C-reactive protein; DNI: delta neutrophil index.

Table 2. Echocardiographic parameters and cardiac biomarkers of patients

\begin{tabular}{|c|c|c|c|c|c|}
\hline \multirow{2}{*}{ Echocardiographic parameter } & \multicolumn{2}{|c|}{$\operatorname{SIC}(n=49)$} & \multicolumn{2}{|c|}{ Non-SIC $(n=259)$} & \multirow{2}{*}{ P-value } \\
\hline & Individual & Value & Individual & Value & \\
\hline Ejection fraction (\%) & 49 & $34.3 \pm 8.4$ & 259 & $64.7 \pm 7.5$ & $<0.001$ \\
\hline $\operatorname{LVEDD~}(\mathrm{mm})$ & 46 & $49.6 \pm 6.0$ & 248 & $43.4 \pm 6.3$ & $<0.001$ \\
\hline LVESD (mm) & 46 & $39.5 \pm 6.4$ & 248 & $28.7 \pm 4.8$ & $<0.001$ \\
\hline Mitral E/e' ratio & 36 & $12.9 \pm 5.5$ & 198 & $12.0 \pm 4.3$ & 0.341 \\
\hline FAC $(\%)$ & 7 & $25.3 \pm 5.3$ & 17 & $24.0 \pm 8.7$ & 0.712 \\
\hline \multicolumn{6}{|l|}{ Cardiac biomarker } \\
\hline NT-proBNP (pg/ml) & 46 & $19,911.2 \pm 18,194.9$ & 233 & $7,940.3 \pm 13,613.9$ & $<0.001$ \\
\hline CK (IU/L) & 49 & $341.7 \pm 491.8$ & 245 & $635.9 \pm 3,711.8$ & 0.235 \\
\hline CK-MB (ng/ml) & 49 & $8.0 \pm 12.8$ & 252 & $11.9 \pm 60.3$ & 0.357 \\
\hline Troponin-T (pg/ml) & 48 & $278.9 \pm 492.7$ & 230 & $104.8 \pm 178.4$ & 0.019 \\
\hline
\end{tabular}

Values are presented as mean \pm standard deviation.

SIC: sepsis-induced cardiomyopathy; LVEDD: left ventricular end-diastolic diameter; LVESD: left ventricular end-systolic diameter; E: early mitral inflow velocity; e': mitral annular early diastolic velocity; FAC: fractional area change; NT-proBNP: N-terminal pro b-type natriuretic peptide; CK: creatine kinase; CK-MB: creatine kinase-muscle/brain.

admission and increased LVEDD were independent risk factors for the development of SIC (Table 3).

\section{Outcomes of SIC}

There was no significant difference between SIC patients and non-SIC patients in 28-day all-cause mortality (24.5\% vs. $26.3 \%$, $\mathrm{P}=0.936)$, length of ICU stay in days $(15.4 \pm 13.8$ vs. $12.4 \pm 20.2$, $\mathrm{P}=0.249)$, ICU mortality ( $26.5 \%$ vs. $24.3 \%, \mathrm{P}=0.882)$, and inhospital mortality ( $36.7 \%$ vs $36.7 \%, \mathrm{P}=1.000$ ) (Table 4). Among 31 patients who survived in the hospital, 25 were discharged home and six were transferred to another hospital. Cause of death for those who died in the hospital were as follows: pri- mary infection-related multiple organ failure $(n=10)$, respiratory failure $(n=3)$, end of life decision $(n=4)$, sudden cardiac $\operatorname{arrest}(\mathrm{n}=1)$.

SIC patients were divided into survivors and non-survivors according to 28-day all-cause mortality, as shown in Table 5. Non-survivors showed higher APACHE II ( $32.1 \pm 8.3$ vs. $24.4 \pm$ 8.9, $\mathrm{P}=0.012)$ and SOFA scores $(11.7 \pm 3.3$ and $9.1 \pm 3.0, \mathrm{P}=0.015)$ than survivors. Non-survivors showed significantly lower platelet counts (54.0; interquartile range [IQR], 29.0-138.5 vs. 155.0; IQR, 76.0-271.0; $\mathrm{P}=0.024)$ and higher DNI (26.1; IQR, 3.0-45.9 vs. 3.2; IQR, 1.1-9.5; $\mathrm{P}=0.049$ ) than survivors. Regarding vital signs on admission, non-survivors showed a higher heart rate 
Table 3. Logistic regression analyses for predictors of sepsis induced cardiomyopathy

\begin{tabular}{|c|c|c|}
\hline Variable & $\mathrm{HR}(95 \% \mathrm{Cl})$ & P-value \\
\hline \multicolumn{3}{|c|}{ Univariate logistic regression analysis } \\
\hline Age & $0.99(0.97-1.02)$ & 0.714 \\
\hline Male sex & $0.73(0.31-1.70)$ & 0.460 \\
\hline Diabetes mellitus & $0.59(0.25-1.38)$ & 0.221 \\
\hline Heart failure & $4.79(0.29-78.86)$ & 0.273 \\
\hline Systolic blood pressure & $0.98(0.96-1.00)$ & 0.027 \\
\hline Heart rate & $1.00(0.98-1.01)$ & 0.748 \\
\hline CRP & $1.00(1.00-1.01)$ & 0.088 \\
\hline Procalcitonin & $1.01(0.99-1.02)$ & 0.357 \\
\hline DNI & $1.00(0.97-1.02)$ & 0.821 \\
\hline Lactate & $0.93(0.83-1.05)$ & 0.264 \\
\hline Albumin & $0.43(0.20-0.94)$ & 0.035 \\
\hline Blood culture (+) & $1.28(0.57-2.87)$ & 0.553 \\
\hline NT-proBNP/1000 & $1.03(1.01-1.06)$ & 0.001 \\
\hline Troponin T/10 & $1.03(1.01-1.06)$ & 0.007 \\
\hline LVEDD & $1.19(1.09-1.28)$ & $<0.001$ \\
\hline E/e' & $1.05(0.97-1.14)$ & 0.195 \\
\hline \multicolumn{3}{|c|}{ Multivariate logistic regression analysis } \\
\hline Systolic blood pressure & $0.96(0.93-0.99)$ & 0.007 \\
\hline Albumin & $0.38(0.14-1.07)$ & 0.066 \\
\hline NT-proBNP/1000 & $1.04(0.99-1.04)$ & 0.296 \\
\hline Troponin T/10 & $1.01(1.00-1.03)$ & 0.068 \\
\hline LVEDD & $1.24(1.13-1.37)$ & $<0.001$ \\
\hline
\end{tabular}

HR: hazard ratio; Cl: confidential interval; CRP: c-reactive protein; DNI: delta neutrophil index; NT-proBNP: N-terminal pro b-type natriuretic peptide; LVEDD: left ventricular end-diastolic diameter; E: early mitral inflow velocity; e': mitral annular early diastolic velocity.

than survivors $(134.3 \pm 25.8$ vs. $101.5 \pm 25.2, \mathrm{P}<0.001)$. Cox hazard proportional regression analysis for 28-day mortality in SIC patients revealed that APACHE II (hazard ratio [HR], 1.10; 95\% confidential interval [CI], 1.02 to $1.18 ; \mathrm{P}=0.009$ ) and DNI (HR, 1.02; 95\% CI, 1.00 to 1.08; $\mathrm{P}=0.026$ ) were independent risk factors, while TEE parameters and cardiac biomarkers did not show statistically significant correlations with 28day mortality. Cox hazard proportional regression analysis for 28-day mortality in sepsis patients as a whole revealed that APACHE II (HR, 1.04; 95\% CI, 1.01 to 1.07; $\mathrm{P}=0.004)$, DNI (HR, $1.02 ; 95 \% \mathrm{CI}, 1.00$ to $1.03 ; \mathrm{P}=0.044)$, and lactate $(\mathrm{HR}, 1.07 ; 95 \%$ CI, 1.02 to 1.13; $\mathrm{P}=0.007$ ) were independent risk factors (Table 6). These variables are known to represent sepsis severity [1214]. This suggests that sepsis severity, rather than TTE parameters and cardiac biomarkers, has the most important effect
Table 4. Outcome of sepsis induced cardiomyopathy

\begin{tabular}{lcccc}
\hline Variable & $\begin{array}{c}\text { Total } \\
(\mathrm{n}=308)\end{array}$ & $\begin{array}{c}\text { SIC } \\
(\mathrm{n}=49)\end{array}$ & $\begin{array}{c}\text { Non-SIC } \\
(\mathrm{n}=259)\end{array}$ & P-value \\
\hline 28-Day mortality & $80(26.0)$ & $12(24.5)$ & $68(26.3)$ & 0.936 \\
In-hospital mortality & $113(36.7)$ & $18(36.7)$ & $95(36.7)$ & 1.000 \\
ICU mortality & $76(24.7)$ & $13(26.5)$ & $63(24.3)$ & 0.882 \\
Length of ICU stay (day) & $13.2 \pm 19.3$ & $15.4 \pm 13.8$ & $12.7 \pm 20.2$ & 0.249 \\
\hline
\end{tabular}

Values are presented as number (\%) or mean \pm standard deviation. SIC: sepsis-induced cardiomyopathy; ICU: intensive care unit.

on SIC mortality.

Using the definition of SIC delineated in this study, patients with reversible $\mathrm{LV}$ function deterioration due to sepsis were compared with non-SIC patients. Of 83 patients who showed LV function deterioration, 15 patients with persistent LV dysfunction in follow-up TTE and 19 patients without follow-up TTE were excluded. The mortality outcomes of these two excluded groups are shown in Figure 1. While patients with persistent LV dysfunction revealed similar mortality outcomes as SIC and non-SIC patients, the group of patients without follow-up TTE showed a significantly poorer mortality outcome (28-day mortality, 73.7\%). Follow-up TTE data were not available within 14 days from the first TTE in this group of patients for the following reasons. Eight patients (42.1\%) died within 72 hours after ICU admission, end-of-life decisions were made for six patients (31.6\%) mostly due to terminal underlying diseases (e.g., cancer), two patients were transferred to other hospitals, and three patients (15.8\%) recovered from sepsis.

\section{DISCUSSION}

In this study, we demonstrated the clinical features, predictors, and survival outcomes of SIC. Low systolic blood pressure and increased LVEDD were revealed to be independent predictors of SIC. There was no significant difference between SIC and sepsis patients as a whole in terms of 28-day all-cause mortality. However, high APACHE II scores and DNI, which reflect sepsis severity, were independent risk factors for 28day all-cause mortality in SIC patients.

To define SIC, we used a definition commonly applied in previous studies $[2,7,10]$. In these studies, the inclusion of preexisting cardiac diseases was controversial. In some studies, patients with pre-existing cardiac disease were excluded to reduce false-positive errors in detecting SIC [4,5,10,15-17]. However, those with pre-existing cardiac disease should be included and evaluated if this condition alters the risk of SIC 
Table 5. Baseline characteristics of sepsis-induced cardiomyopathy patients according to 28-day all-cause mortality

\begin{tabular}{|c|c|c|c|}
\hline Characteristics & Survivor $(n=37)$ & Non-survivor $(n=12)$ & P-value \\
\hline Age (yr) & $65.0 \pm 11.2$ & $65.3 \pm 11.7$ & 0.935 \\
\hline Male sex & $24(64.9)$ & $8(66.7)$ & 1.000 \\
\hline $\mathrm{BMI}\left(\mathrm{kg} / \mathrm{m}^{2}\right)$ & $22.3 \pm 3.8$ & $22.6 \pm 4.3$ & 0.837 \\
\hline APACHE II & $24.4 \pm 8.9$ & $32.1 \pm 8.3$ & 0.012 \\
\hline SOFA & $9.1 \pm 3.0$ & $11.7 \pm 3.3$ & 0.015 \\
\hline Charlson comorbidity index & $3.0(2.0-4.0)$ & $3.0(2.0-4.5)$ & 0.715 \\
\hline Acute kidney injury & $10(27.0)$ & $4(33.3)$ & 0.721 \\
\hline ARDS & $8(21.6)$ & 0 & 0.173 \\
\hline Mechanical ventilation & $25(67.6)$ & $11(91.7)$ & 0.142 \\
\hline Blood culture (+) & $16(43.2)$ & $6(50.0)$ & 0.940 \\
\hline \multicolumn{4}{|l|}{ Vital sign on admission } \\
\hline Systolic blood pressure $(\mathrm{mm} \mathrm{Hg})$ & $83.7 \pm 18.5$ & $84.7 \pm 18.3$ & 0.876 \\
\hline Mean arterial pressure $(\mathrm{mm} \mathrm{Hg})$ & $64.8 \pm 15.1$ & $63.1 \pm 16.7$ & 0.746 \\
\hline Heart rate (bpm) & $101.5 \pm 25.2$ & $134.3 \pm 25.8$ & $<0.001$ \\
\hline \multicolumn{4}{|l|}{ Use of vasopressor } \\
\hline Norepinephrine & $32(86.5)$ & $12(100.0)$ & 0.427 \\
\hline Dobutamine & $2(5.4)$ & 0 & 1.000 \\
\hline \multicolumn{4}{|l|}{ Laboratory parameter } \\
\hline $\operatorname{WBC}\left(10^{3} / \mu \mid\right)$ & $10.3(7.0-17.1)$ & $9.4(3.0-14.7)$ & 0.429 \\
\hline Platelet $\left(10^{3} / \mu \mathrm{l}\right)$ & $155.0(76.0-271.0)$ & $54.0(29.0-138.5)$ & 0.024 \\
\hline Serum albumin (g/dl) & $2.4 \pm 0.6$ & $2.4 \pm 0.5$ & 0.872 \\
\hline Serum bilirubin (mg/dl) & $0.7(0.4-1.1)$ & $1.0(0.4-1.9)$ & 0.470 \\
\hline Serum creatinine (mg/dl) & $1.3(0.9-1.9)$ & $2.0(1.2-3.6)$ & 0.212 \\
\hline Lactate (mmol/L) & $2.5(1.6-3.8)$ & $4.0(2.0-7.6)$ & 0.089 \\
\hline CRP & $179.1(100.7-257.4)$ & $185.9(49.5-343.2)$ & 1.000 \\
\hline Procalcitonin (ng/ml) & $5.1(1.3-30.3)$ & $5.6(0.8-40.6)$ & 0.917 \\
\hline DNI (\%) & $3.2(1.1-9.5)$ & $26.1(3.0-45.9)$ & 0.049 \\
\hline \multicolumn{4}{|l|}{ Echocardiographic finding } \\
\hline Ejection fraction (\%) & $34.9 \pm 8.7$ & $32.6 \pm 7.5$ & 0.410 \\
\hline LVEDD (mm) & $50.2 \pm 5.1(n=35)$ & $47.6 \pm 8.1(n=11)$ & 0.339 \\
\hline LVESD (mm) & $39.7 \pm 5.8(n=35)$ & $39.1 \pm 8.2(n=11)$ & 0.800 \\
\hline Mitral E/e' ratio & $12.9 \pm 4.8(n=31)$ & $13.2 \pm 10.0(n=5)$ & 0.943 \\
\hline \multicolumn{4}{|l|}{ Cardiac biomarker } \\
\hline NT-proBNP (pg/ml) & $11,983.0(7,514.5-30,442.0)(n=35)$ & $22,073.0(3,705.5-35,000.0)(n=11)$ & 0.857 \\
\hline CK (IU/L) & $76.0(35.0-331.0)$ & $171.0(59.0-688.0)$ & 0.231 \\
\hline CK-MB (ng/ml) & $4.2(2.0-7.2)$ & $2.1(1.5-5.3)$ & 0.236 \\
\hline Troponin-T (pg/ml) & $156.0(47.0-318.0)$ & $108.0(62.5-304.0)(n=11)$ & 0.980 \\
\hline
\end{tabular}

Values are presented as mean \pm standard deviation, number (\%), or median (interquartile range).

BMI: body mass index; APACHE: Acute Physiology and Chronic Health Evaluation; SOFA: Sequential Organ Failure Assessment; ARDS: acute respiratory distress syndrome; WBC: white blood cell; CRP: C-reactive protein; DNI: delta neutrophil index; LVEDD: left ventricular end-diastolic diameter; LVESD: left ventricular end-systolic diameter; E: early mitral inflow velocity; e': mitral annular early diastolic velocity; NT-proBNP: N-terminal pro b-type natriuretic peptide; CK: creatine kinase; CK-MB: creatine kinase-muscle/brain. 
Table 6. Cox proportional hazard regression analysis for 28-day mortality in SIC patients and sepsis patients as a whole

\begin{tabular}{|c|c|c|c|c|}
\hline \multirow{2}{*}{ Variable } & \multicolumn{2}{|l|}{ Univariate } & \multicolumn{2}{|c|}{ Multivariate } \\
\hline & HR $(95 \% \mathrm{Cl})$ & P-value & $\mathrm{HR}(95 \% \mathrm{Cl})$ & P-value \\
\hline \multicolumn{5}{|l|}{ SIC patient } \\
\hline APACHE II & $1.08(1.02-1.14)$ & 0.006 & $1.10(1.02-1.18)$ & 0.009 \\
\hline DNI & $1.03(0.01-2.21)$ & 0.027 & $1.02(1.00-1.08)$ & 0.026 \\
\hline Lactate & $1.12(1.01-1.24)$ & 0.036 & $1.12(0.74-1.07)$ & 0.210 \\
\hline EF & $0.97(0.91-1.04)$ & 0.381 & & \\
\hline LVEDD & $0.94(0.85-1.04)$ & 0.231 & & \\
\hline \multicolumn{5}{|c|}{ Sepsis patients as a whole } \\
\hline APACHE II & $1.06(1.03-1.09)$ & $<0.001$ & $1.04(1.01-1.07)$ & 0.004 \\
\hline DNI & $1.03(0.01-1.04)$ & $<0.001$ & $1.02(1.00-1.03)$ & 0.044 \\
\hline Lactate & $1.12(1.07-1.16)$ & $<0.001$ & $1.07(1.02-1.13)$ & 0.007 \\
\hline EF & $1.01(0.99-1.03)$ & 0.321 & & \\
\hline LVEDD & $0.96(0.93-0.98)$ & 0.008 & $0.97(0.93-1.01)$ & 0.118 \\
\hline SIC & $0.89(0.0 .48-1.65)$ & 0.723 & $0.88(0.43-1.74)$ & 0.688 \\
\hline
\end{tabular}

SIC: sepsis-induced cardiomyopathy; HR: hazard ratio; $\mathrm{Cl}$ : confidential interval; APACHE: Acute Physiology and Chronic Health Evaluation; DNI: delta neutrophil index; EF: ejection fraction; LVEDD: left ventricular enddiastolic diameter.

development and survival. Our study revealed that neither heart failure nor coronary artery disease was a risk factor for SIC development or mortality.

Recently, several studies have indicated differences in the pathophysiology of SIC and stress-induced cardiomyopathy, also known as Takotsubo cardiomyopathy, in which sepsis is the source of stress $[7,9,10]$. Endotoxins, inflammatory cytokines, and nitric oxides are major contributors to the development of SIC $[18,19]$. In contrast, elevated catecholamine release is a key contributor to the development of Takotsubo cardiomyopathy [20]. However, it is difficult to distinguish these two diseases based on clinical parameters. Previous studies that distinguished SIC and Takotsubo cardiomyopathy used the criterion of typical apical ballooning in TTE. However, Takotsubo cardiomyopathy has both apical ballooning type and atypical type, which are difficult to discriminate from SIC based on TTE findings $[20,21]$. Thus, we proposed that SIC is a syndrome characterized by reversible LV dysfunction and includes Takotsubo cardiomyopathy caused by sepsis. Endotoxins, inflammatory cytokines, nitric oxides, and catecholamine are all considered associated with SIC development.

Echocardiography revealed that LVEDD was significantly higher in SIC patients than in non-SIC patients. Diastolic ventricle size increased to compensate for decreased systolic contractility. After fluid resuscitation, stroke volume can recover while EF is temporarily decreased due to increased LVEDD. Based on this pathophysiology, the importance of diastolic dysfunction rather than systolic function has been emphasized recently with reports of correlation between a lower e', higher $\mathrm{E} / \mathrm{e}^{\prime}$ ratio, and mortality in sepsis patients $[19,22]$. However, in this study, the LVEDD and E/e' ratio did not show significant differences between survivors and non-survivors. This might be due to missing TTE parameters, especially in non-survivors.

In this study, low systolic blood pressure upon ICU admission and increased LVEDD were considered predictors of SIC development. Low systolic blood pressure resulting in inadequate coronary blood flow has been proposed as a mechanism of SIC based on animal studies [23]. Dilated LVEDD is diastolic compliance to decreased systolic function [19]. The predictors of SIC identified in this study are discrepant from those previously identified, probably due to the small sample size of each study and the use of discordant definitions $[7,10,24]$.

In SIC patients, tachycardia on ICU admission was significantly more common among non-survivors than survivors. This is explained by adapting to insufficient diastolic filling to increase stroke volume. In the Cox proportional hazard regression analysis of 28-day mortality, APACHE II score and DNI were revealed as independent risk factors for mortality in SIC patients. Both APACHE II and DNI are markers of sepsis severity. DNI is a novel biomarker that reflects the number of circulating granulocytes in blood and correlates with sepsis severity in critically ill patients $[11,12,25,26]$. This suggests that sepsis severity has a greater effect on SIC prognosis than TEE parameters and cardiac biomarkers.

Among the 83 patients who showed LV dysfunction, 19 had no follow-up TTE. This group of patients showed significantly poorer mortality outcome, suggesting that the cardiac function of these 19 patients deteriorated due to sepsis and they usually did not recover, resulting in mortality. This may imply that this group of patients had the same pathophysiology as patients with SIC development and poor outcome. Due to the small number of these patients, the characteristics of persistent $\mathrm{LV}$ dysfunction could not be analyzed in detail in this group with no follow-up TTE. Further studies should be performed to clarify the characteristics of these patients.

This study had several limitations. First, detailed information regarding fluid resuscitation and vasopressor initiation were not available. These factors strongly affect sepsis patient prognosis. However, the study was performed in a single ICU of a tertiary university hospital in which sepsis management, including fluid resuscitation and vasopressor initiation, was generally performed according to the more recent sepsis-3 
guidelines. Second, baseline TTE and TTE within 48 hours were not available in $8.7 \%$ of the initial population. This selection bias may have influenced the final results. Third, TTE parameters were not documented equally among patients and laboratory data. For example, inflammatory cytokines and catecholamines were not available due to the retrospective nature of this study. Lastly, this was a single center, retrospective study, which could affect the generalizability of results. Further prospective studies are needed to validate the results of this study.

In conclusion, this study identified predictors and outcome of SIC with inclusion of pre-existing cardiac diseases and Takotsubo cardiomyopathy. We found that low systolic blood pressure and dilated LVEDD were SIC predictors. The prognosis of SIC was affected by sepsis severity rather than cardiac function. Reversible SIC does not increase mortality risk compared to non-SIC. Further studies are needed on sepsis patients with persistent LV dysfunction and on patients whose LV function could not be restored, resulting in death.

\section{CONFLICT OF INTEREST}

No potential conflict of interest relevant to this article was reported.

\section{ORCID}

Myung Jin Song

Sang Hoon Lee

Ah Young Leem

Song Yee Kim

Kyung Soo Chung

Eun Young Kim

Ji Ye Jung

Young Ae Kang

Young Sam Kim

Joon Chang

Moo Suk Park https://orcid.org/0000-0003-2218-8959

https://orcid.org/0000-0002-7706-5318

https://orcid.org/0000-0001-5165-3704

https://orcid.org/0000-0001-8627-486X

https://orcid.org/0000-0003-1604-8730

https://orcid.org/0000-0002-3281-5744

https://orcid.org/0000-0003-1589-4142

https://orcid.org/0000-0002-7783-5271

https://orcid.org/0000-0001-9656-8482

https://orcid.org/0000-0003-4542-6841

https://orcid.org/0000-0003-0820-7615

\section{AUTHOR CONTRIBUTIONS}

Conceptualization: MJS, MSP. Data curation, Formal analysis, \& Methodology: all authors. Project administration, Visualization, \& Writing-original draft: MJS, MSP. Writing-review \& editing: all authors.

\section{REFERENCES}

1. Singer M, Deutschman CS, Seymour CW, Shankar-Hari M, Annane D, Bauer M, et al. The Third International Consensus Definitions for Sepsis and Septic Shock(Sepsis-3). JAMA 2016; 315:801-10.

2. Parker MM, Shelhamer JH, Bacharach SL, Green MV, Natanson C, Frederick TM, et al. Profound but reversible myocardial depression in patients with septic shock. Ann Intern Med 1984;100:483-90.

3. Jardin F, Brun-Ney D, Auvert B, Beauchet A, Bourdarias JP. Sepsis-related cardiogenic shock. Crit Care Med 1990;18:105560.

4. Etchecopar-Chevreuil C, François B, Clavel M, Pichon N, Gastinne H, Vignon P. Cardiac morphological and functional changes during early septic shock: a transesophageal echocardiographic study. Intensive Care Med 2008;34:250-6.

5. Vieillard-Baron A, Caille V, Charron C, Belliard G, Page B, Jardin F. Actual incidence of global left ventricular hypokinesia in adult septic shock. Crit Care Med 2008;36:1701-6.

6. Bouhemad B, Nicolas-Robin A, Arbelot C, Arthaud M, Féger F, Rouby JJ. Acute left ventricular dilatation and shock-induced myocardial dysfunction. Crit Care Med 2009;37:441-7.

7. Sato R, Kuriyama A, Takada T, Nasu M, Luthe SK. Prevalence and risk factors of sepsis-induced cardiomyopathy: a retrospective cohort study. Medicine (Baltimore) 2016;95:e5031.

8. Calandra T, Cohen J; International Sepsis Forum Definition of Infection in the ICU Consensus Conference. The international sepsis forum consensus conference on definitions of infection in the intensive care unit. Crit Care Med 2005;33:1538-48.

9. Sato R, Nasu M. A review of sepsis-induced cardiomyopathy. J Intensive Care 2015;3:48.

10. Jeong HS, Lee TH, Bang CH, Kim JH, Hong SJ. Risk factors and outcomes of sepsis-induced myocardial dysfunction and stress-induced cardiomyopathy in sepsis or septic shock: a comparative retrospective study. Medicine (Baltimore) 2018; 97:e0263.

11. Nahm CH, Choi JW, Lee J. Delta neutrophil index in automated immature granulocyte counts for assessing disease severity of patients with sepsis. Ann Clin Lab Sci 2008;38:241-6.

12. Park BH, Kang YA, Park MS, Jung WJ, Lee SH, Lee SK, et al. Delta neutrophil index as an early marker of disease severity in critically ill patients with sepsis. BMC Infect Dis 2011;11:299.

13. Kruse JA, Thill-Baharozian MC, Carlson RW. Comparison of clinical assessment with APACHE II for predicting mortality risk in patients admitted to a medical intensive care unit. JAMA 1988;260:1739-42. 
14. Mikkelsen ME, Miltiades AN, Gaieski DF, Goyal M, Fuchs BD, Shah CV, et al. Serum lactate is associated with mortality in severe sepsis independent of organ failure and shock. Crit Care Med 2009;37:1670-7.

15. Furian T, Aguiar C, Prado K, Ribeiro RV, Becker L, Martinelli $\mathrm{N}$, et al. Ventricular dysfunction and dilation in severe sepsis and septic shock: relation to endothelial function and mortality. J Crit Care 2012;27:319.

16. Pulido JN, Afessa B, Masaki M, Yuasa T, Gillespie S, Herasevich V, et al. Clinical spectrum, frequency, and significance of myocardial dysfunction in severe sepsis and septic shock. Mayo Clin Proc 2012;87:620-8.

17. Weng L, Liu YT, Du B, Zhou JF, Guo XX, Peng JM, et al. The prognostic value of left ventricular systolic function measured by tissue Doppler imaging in septic shock. Crit Care 2012;16:R71.

18. Liu YC, Yu MM, Shou ST, Chai YF. Sepsis-induced cardiomyopathy: mechanisms and treatments. Front Immunol 2017;8: 1021.

19. Walley KR. Sepsis-induced myocardial dysfunction. Curr Opin Crit Care 2018;24:292-9.
20. Boland TA, Lee VH, Bleck TP. Stress-induced cardiomyopathy. Crit Care Med 2015;43:686-93.

21. Templin C, Ghadri JR, Diekmann J, Napp LC, Bataiosu DR, Jaguszewski M, et al. Clinical features and outcomes of Takotsubo (Stress) cardiomyopathy. N Engl J Med 2015;373:929-38.

22. Kakihana Y, Ito T, Nakahara M, Yamaguchi K, Yasuda T. Sepsis-induced myocardial dysfunction: pathophysiology and management. J Intensive Care 2016;4:22.

23. Bruni FD, Komwatana P, Soulsby ME, Hess ML. Endotoxin and myocardial failure: role of the myofibril and venous return. Am J Physiol 1978;235:H150-6.

24. Mehta NJ, Khan IA, Gupta V, Jani K, Gowda RM, Smith PR. Cardiac troponin I predicts myocardial dysfunction and adverse outcome in septic shock. Int J Cardiol 2004;95:13-7.

25. Kim HW, Yoon JH, Jin SJ, Kim SB, Ku NS, Jeong SJ, et al. Delta neutrophil index as a prognostic marker of early mortality in gram negative bacteremia. Infect Chemother 2014;46:94-102.

26. Yune HY, Chung SP, Park YS, Chung HS, Lee HS, Lee JW, et al. Delta neutrophil index as a promising prognostic marker in out of hospital cardiac arrest. PLoS One 2015;10:e0120677. 